\title{
Efficient joint identification and fluted segment modelling of shrink-fit tool assemblies by updating extended tool models
}

\author{
Christian Brecher ${ }^{1} \cdot$ Prateek Chavan $^{1}\left[\right.$. Marcel Fey ${ }^{1}$
}

Received: 29 August 2020 / Accepted: 3 November 2020 / Published online: 23 December 2020

(c) The Author(s) 2020

\begin{abstract}
In milling, the dynamic behavior of the tool center point is crucial for estimating surface quality of the workpiece as well as the process stability behavior. Experimental-analytical receptance coupling can be used for predicting the tool tip dynamics but requires accurate analytical modelling of the holder-tool assembly. This includes the reliable identification of the holder-tool joint properties as well as the correct modelling of the fluted segment of end mills. However, the modelling effort associated with accurately representing the dynamic behavior of the fluted segment is significant. In addition, the joint identification requires a reference tool tip frequency response function of the tool assembly clamped in the machine spindle. This is inefficient and can also lead to incorrect estimation of joint properties. This paper provides an efficient method for joint identification and fluted section modelling using an offline, free-free excitation approach. The objective of this paper is to enable a direct comparison of the dynamic behavior of the freely constrained analytical tool assembly model with that of the real freely constrained tool assembly. The comparison of displacement to force frequency response at certain points on the tool assembly allows for the identification of tool model parameters such as the joint properties and effective diameter of the fluted segment. The comparability is realized by extending the analytical holder-tool beam model to include the receptance model of the standard spindle-holder interface. In this study, as an example, a thermal shrink-fit holder-tool beam model is extended to include an HSK-A63 interface. Subsequently, frequency response functions at two points on the real freely constrained tool assembly are measured in order to identify the joint stiffness and effective diameter of the fluted segment using the corresponding proposed formulations. The updated holder-tool model is then coupled with a 4-axis milling machine and validated. Despite the reduced modelling effort, a good prediction accuracy could be achieved for different holder-tool combinations.
\end{abstract}

Keywords Tool coupling $\cdot$ Experimental dynamic substructuring $\cdot$ Vibration prediction $\cdot$ Substructure coupling

\section{Introduction}

The relative dynamic compliance behavior between the workpiece and tool determines significantly the allowable material removal rate without instability, surface quality as well as achievable form accuracy [3]. This dynamic behavior is in turn determined by the compliance behavior of all components and joints lying within the force flux of the process force. This includes the cutting tool assembly, main spindle, machine column and bed, feed drives, workpiece

Prateek Chavan

p.chavan@wzl.rwth-aachen.de

1 Laboratory for Machine Tools and Production Engineering (WZL) of RWTH Aachen University, Aachen, NRW, Germany table, workpiece itself, etc. Among these elements, the milling tool is frequently changed as per cutting requirements, thereby changing the dynamic behavior with each variation of tool dimensions. Since the measurement of each tool tip frequency response function (FRF) in the machine is not efficient, the method of frequency-based experimental-analytical receptance coupling has been proposed in literature for an efficient prediction.

In [16], the Receptance Coupling Substructuring Analysis (RCSA) approach was first proposed for tool coupling where the spindle-side displacement to force receptance was obtained experimentally and the milling tool was simplified as an analytical Euler-Bernoullie beam element. Although the joint between the tool holder and tool had both translational and rotational degrees of freedom (DOF), the tool and the spindle-side receptances were considered rotationally 
rigid. Since then, several studies have improved upon the RCSA approach. Park et al. proposed a formulation which included receptances not only of translational DOFs but also for rotational DOFs [14]. The receptances of the spindle and tool holder were obtained experimentally by impact tests on carbide blanks inserted in the holder. They reported a significant improvement in the prediction accuracy by including the rotational DOFs. Later, Namazi et al. extended Park's method in order to identify the complete receptance matrix at the tool holder flange (or standard spindle-holder interface) by inverse receptance coupling [10]. It was also shown that modelling the holder-tool assembly using Timoshenko beam elements led to accurate prediction of the tool tip FRF even at higher frequencies. Although the Timoshenko beam theory provides a realistic representation of the behavior of beams by considering rotational inertia and shear effects, the continuous multi-segment beam modelling requires a solution of a determinant of a high order matrix. The difficulties associated with solving a large eigenvalue problem are overcome in [4]. Here, the eigenvalue problem is solved for individual freely constrained Timoshenko beam elements or segments. The model of the tool assembly is obtained by endto-end receptance coupling of the single-segment beams. The resonance frequencies predicted by this method showed a good correspondence with those calculated by an Finite Element (FE) model without being as computationally expensive. Although computational efficiency has improved since then, the method provides a viable alternative for rapid and accurate modelling of beams without requiring $\mathrm{CAD}$ and $\mathrm{FE}$ tool models and corresponding modelling software packages.

Two important challenges, which limit the accuracy of tool tip FRF predictions, are: (i) the accurate modelling and identification of the holder and tool joint properties and (ii) the accurate modelling of the complex fluted segment of the shaft mill.

The fluted segment of end mills has, depending on the number of teeth and helix angle, a complex cross-section, which varies along its length. Kops and Vo in [7] used a cylindrical beam with an equivalent diameter to represent the static stiffness behavior of an end mill. The variation of the area moment of inertia along the radial direction was ignored. They found that an equivalent diameter of $80 \%$ of the tool diameter was suitable for representing the static bending behavior of the 2- and 4-fluted end mills used in the study. Subsequently, other authors have used the simplification of $80 \%$ of tool diameter to represent the fluted segment for receptance coupling [8,9]. Later, Kivanc and Budak extended Kops and Vo's method by including the effect of the flute arcs on the area moment of inertia and also by considering the moment of inertia in two orthogonal directions [6]. In [11], the fluted segment was divided into multiple segments and the area moment of inertia for the tool-end segment was first calculated for the corresponding cross-section using Kivanc and Budak's method. Then, the upper segments were rotated depending on the helix angle and the inertia about the fixed orthogonal coordinate system was calculated. A limitation of the methods presented in the literature is that an exact geometry of the flutes is not readily available and even if it is available, the modelling of each end mill geometry is effortful and requires modelling expertise that might not be available in a workshop environment. The simplification of the equivalent diameter being $80 \%$ of the fluted segment diameter gives a rough engineering estimate but cannot be satisfactorily representative for all combinations of number of teeth, helix angle, length of segments, cooling channels, etc.

The contact stiffness and damping between a tool-holder and tool along its clamped length is difficult to obtain a priori. Several approaches are available in literature for modelling this contact stiffness and damping. A number of authors proposed using a spring-damper element acting between the holder and overhanging tool to represent this joint $[6,11,13$, 18]. Such an element provides additional compliance and equivalent viscous damping caused by the joint contact stiffness and columbic damping. In [1] the joint properties are represented not as a single spring-damper element but rather as an elastic layer along the clamped length. This allows for taking into consideration the variation of normal pressure and stiffness along the clamped length as well as surface roughness. Schmitz et al. also presented a realistic model of the joint by including multiple connections between the holder and the part of the tool within the holder [20,21]. [16] proposed an approach to identify directly the holdertool joint FRFs at multiple points along the length of the holder using inverse RCSA. Due to the identification of multiple connection points at the holder, the effect of rotation is included implicitly and hence the rotational compliances were ignored. An alternate approach for shrink-fit holder joint modelling based on Hertzian contact theory and fractal geometry was developed by Liao et al. [8]. Here the contact stiffness is assumed to be a function of elastic deformation of micro-asperities in contact between the two surfaces.

Irrespective of the method employed for modelling the joint, the parameters of the joint model are obtained in majority of approaches by comparing the predicted tool tip FRF with an experimentally obtained tool tip FRF of the tool clamped in a machine spindle $[1,6,13,15,21]$. Subsequently, the error between prediction and measurement is minimized by varying the joint parameters (usually translational, rotational stiffness and equivalent viscous damping factors). One disadvantage of using the tip FRF in the clamped state as a reference is that the analytical receptance model of the holder-tool assembly has to be coupled with the experimentally derived spindle-interface receptance matrix to allow a comparison. This means that the errors and uncertainties associated with experimentally obtaining 
the spindle-interface receptance matrix are propagated in the predicted spindle-holder-tool tip FRF. Filiz et al. recognized this issue in [5]. Secondly, using the tip FRF in the spindle can lead to identification of incorrect joint parameters which do represent the dynamic behavior of the tool assembly over a larger frequency range [9]. Another disadvantage is the blocking of the operating time of the machine tool, which is required for conducting the measurements with different tool holders and tools. The experimental identification of holder-tool assembly in an unclamped state (freely constrained) can overcome the above-stated limitations. Recently, Matthias et al. demonstrated the identification of joint parameters between a collet holder and carbide blanks using tool tip FRFs measured in a freely constrained state of the holder-tool assembly [9]. For this, the tool holder, including the standard spindle-holder interface, as well as the carbide blanks were modelled by receptance coupling of uniform cylindrical Timoshenko beam segments. However, the modelling of the interface part of the holder as uniform cylindrical multi-segment beam introduces avoidable modelling errors. Especially since standard interfaces are geometrically complex and may contain clamping groove, orientation notch, manual clamping holes, hollow tapered shank, balancing holes, etc., a beam modelling is insufficient. Also the use of Laser Vibrometer for the free-free displacement (from velocity) measurement resulted in poor signal-noise ratio such that the location of anti-resonances could not be observed clearly. This limits the comparability of the predicted and measured FRF for joint identification.

The aim of this paper is to enable the comparison of the freely constrained FRFs of the tool assembly with an extended tool model such that properties of the model can be updated. For this, the interface-holder-tool assembly is divided into two parts: the standardized spindle-holder interface and the holder-tool subassembly. A one-time freely constrained FE-model of the standardized spindle-holder interface is created for obtaining the receptance matrix at the connection point of the interface. The holder-tool system for different tools is modelled using receptance coupling of Timoshenko beams. Subsequently, the analytical beam model of the holder-tool assembly is extended by coupling the interface end of the holder with the receptance (or compliance) matrix of the interface. This augmentation allows for a direct comparison of analytical calculated FRFs with the FRFs obtained experimentally in freely constrained state. These FRFs can then be used for updating different parameters (complex stiffness, effective beam diameter, etc.) of the analytical holder-tool model. Here, the free-free FRFs are measured with an uniaxial acceleration sensor for ensuring good signal to noise ratio. The mass loading effect of the sensors is subsequently decoupled using the structural modification technique. The paper is divided as follows: first mathematical background is provided and the Extended Tool
Model Updating (ETMU) approach is introduced (Sect. 2). This approach is then implemented and analyzed for a shrink-fit holder (with an HSK-A63 interface) for obtaining the joint parameters for different overhang lengths (Sect. 3). Next, using an appropriate receptance coupling formulation, the effective diameters of the fluted segments of end mills with different number of teeth and helix angle are obtained and the tool tip FRF predictions in a 4-axis machine tool are validated (Sect. 3.2). The estimation of effective diameter based on measurement of the freely constrained tool assembly should allow for a realistic approximation of the key dynamic behavior without extensive modelling effort.

\section{Extended tool model updating (ETMU) approach}

Changeable tool system assemblies used in milling and drilling spindles consist of the cutting tool, the tool holder and the standard spindle-holder interface. Depending on the type of automatic tool changer and spindle, different standard spindle-holder interfaces can be used, for example, Morse taper (DIN 228), SK (DIN ISO 7388), HSK (DIN 69,893), TS (DIN ISO 26,622), etc. This normed geometry and shape of the interface is constant for a particular spindle. Hence, the tool assembly model is divided into two parts: the standard interface and the holder-tool assembly. The interface geometry may contain complex groves, varying inner and outer diameters, taper angle, balancing holes, threaded holes, etc. These features cannot be modelled with sufficient accuracy using beam models. Hence, a one-time quadratic tetrahedral FE model of the interface in an unconstrained state is created and the compliance matrices at the holder end are first calculated. An implementation of the proposed approach for a thermal shrink-fit tool holder with an HSKA63 interface is shown as a schematic representation in Fig. 1. Although the HSK interface itself is dynamically balanced, the geometric asymmetry will result in a slightly varying dynamic behavior in the orthogonal $x$ and $y$ directions. Thus, forces $f_{x}, f_{y}$ and moments $M_{x}, M_{y}$ at the master node of the multi-point constraint (MPC) are applied and the corresponding translational $x, y$ and rotational displacements $\theta_{x}, \theta_{y}$ are recorded. The resulting direct receptance matrices of the interface at point 1 in the orthogonal directions are,

$$
\begin{gathered}
\boldsymbol{G}_{11, x}^{I}=\left[\begin{array}{ll}
H_{11, x}^{I} & N_{11, x}^{I} \\
L_{11, x}^{I} & P_{11, x}^{I}
\end{array}\right]=\left[\begin{array}{cc}
\frac{x_{1}^{I}}{F_{1 x}^{I}} & \frac{x_{1}^{I}}{M_{11}^{I}} \\
\frac{\theta_{1 y}^{I}}{F_{1 x}^{I}} & \frac{\theta_{1 y}^{y}}{M_{1 y}^{I}}
\end{array}\right] ; \\
\boldsymbol{G}_{11, y}^{I}=\left[\begin{array}{ll}
H_{11, y}^{I} & N_{11, y}^{I} \\
L_{11, y}^{I} & P_{11, y}^{I}
\end{array}\right]=\left[\begin{array}{ll}
\frac{y_{1}^{I}}{F_{1 y}^{I}} & \frac{y_{1}^{I}}{M_{1 x}^{I}} \\
\frac{\theta_{1 x}^{I}}{F_{1 y}^{I}} & \frac{\theta_{1 x}^{I}}{M_{1 x}^{I}}
\end{array}\right]
\end{gathered}
$$



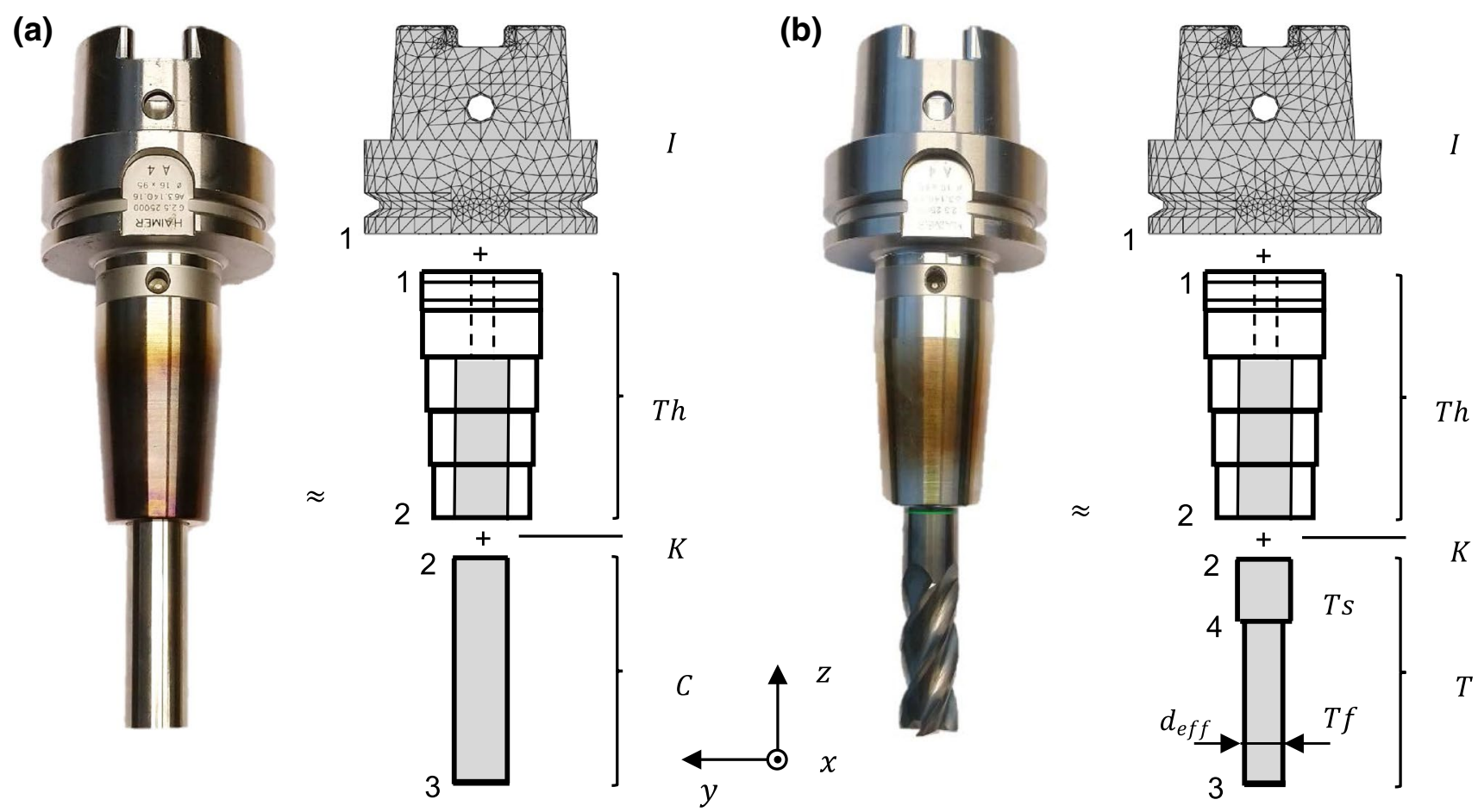

Fig. 1 Schematic illustration of proposed approach for (a) joint identification and (b) for estimation of effective diameter of the fluted segment

For demonstration, coupling calculations are considered only in the $x$ direction in this study. The analytical model of the holder-tool assembly is created by dividing it into multiple uniform cylindrical beam segments. The receptances at the ends of the 2D beam segments are obtained by solving the characteristic equation of the freely constrained Timoshenko beam as proposed by [4] based on the method for generalized end conditions presented by Aristizabal-Ochoa [2]. The end-to-end rigid coupling of the receptances of the individual segments gives the receptance matrix of the complete tool holder including the inserted tool: $\boldsymbol{G}_{11}^{T h}, \boldsymbol{G}_{12}^{T h}, \boldsymbol{G}_{21}^{T h}$ and $\boldsymbol{G}_{22}^{T h}$. Now the model of the holder can be extended by rigidly coupling the receptance matrix of the spindle-holder interface $(I)$. Thus, the compliance matrix at the free end of the extended holder model is given by

$\boldsymbol{G}_{22}^{I T h}=\boldsymbol{G}_{22}^{T h}+\boldsymbol{G}_{21}^{T h}\left(\boldsymbol{G}_{11}^{T h}+\boldsymbol{G}_{11}^{I}\right)^{-1} \boldsymbol{G}_{12}^{T h}$

In a second step, for the purpose of joint stiffness identification, a one-segment beam is rigidly coupled to the freeend of the holder and the free-end dynamics are compared with the corresponding measurement (Fig. 1a). In a third step, for the purpose of estimating the effective diameter of the fluted segment, a two segment beam is coupled flexibly with the extended holder assembly using the previously identified joint stiffness (Fig. 1b). An overview of the proposed method is presented in the form of a flow-chart in Fig. 2a.

\subsection{Joint parameter identification}

For identifying the joint parameters between the shrink-fit holder and tool, a carbide blank of the same length as the tool is inserted in the holder with the same insertion depth. In this paper, the holder-tool joint is represented by a springdamper element with a translational and a rotational degree of freedom. However, the proposed approach can be applied for identifying the parameters of other joint models such has the uniform elastic layer or multiple point joint model. For a shrink-fit joint, a mass-less spring-damper element can be assumed because the masses of the substructures are several orders of magnitude larger than the mass representing the joint. For such an element, the complex stiffness matrix $\boldsymbol{K}$ is given by,

$\boldsymbol{K}=\left[\begin{array}{cc}k_{x f}+i \omega c_{x f} & 0 \\ 0 & k_{\theta y M}+i \omega c_{\theta y M}\end{array}\right]$

where $k_{x f}$ corresponds to the translational stiffness and $i \omega c_{x f}$ to the equivalent translational frequency-dependent damping. Similarly, $k_{\theta y M}, c_{\theta y M}$ correspond to the rotational stiffness and damping factor. The cross-stiffness and damping terms are ignored for simplicity. The elastic coupling of the extended holder $(I T h)$ with the carbide blank $(C)$ is achieved by the following coupling equation and gives the compliance matrix at point 3 of the extended tool assembly IThC: 
(a)

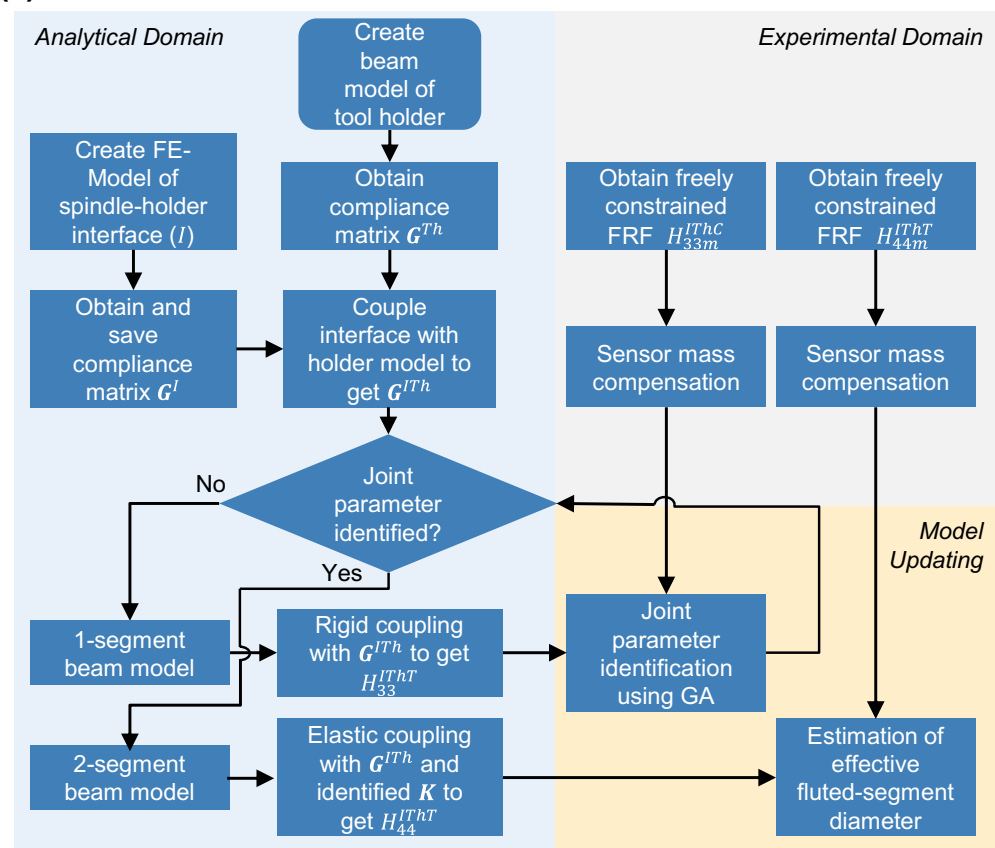

(b)

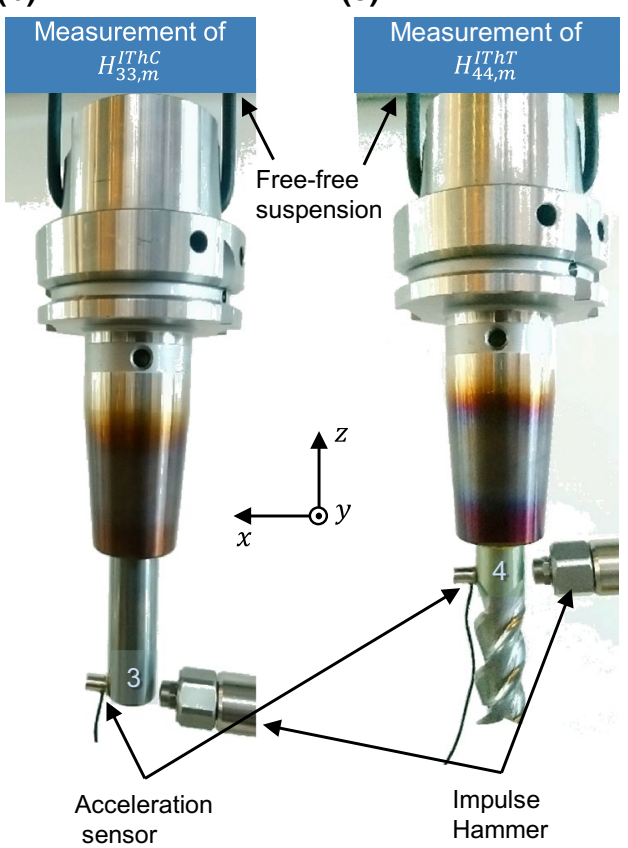

Fig. 2 a Proposed approach for model updating; experimental setup for measuring $\mathbf{b} \mathrm{H}_{33 \mathrm{~m}}^{\mathrm{IThC}}$ and $\mathbf{c} \mathrm{H}_{44 \mathrm{~m}}^{\mathrm{IThT}}$

$\left[\begin{array}{cc}H_{33}^{I T h C} & N_{33}^{I T h C} \\ L_{33}^{I T h C} & P_{33}^{I T h C}\end{array}\right]=\boldsymbol{G}_{33}^{I T h C}=\boldsymbol{G}_{33}^{C}+\boldsymbol{G}_{32}^{C}\left(\boldsymbol{G}_{22}^{I T h}+\boldsymbol{G}_{22}^{C}+\boldsymbol{K}^{-1}\right)^{-1} \boldsymbol{G}_{23}^{C}$

Considering only the displacement to force FRF,
Since the joint parameters are initially unknown, the displacement to force FRF $H_{33}^{I T h C}$ is calculated without the stiffness term. The corresponding FRF of the freely constrained tool assembly $H_{33, m}^{I T h C}$ is obtained using the setup

$H_{33}^{I T h C}=H_{33}^{C}+\left[\begin{array}{ll}H_{32}^{C} & L_{32}^{C}\end{array}\right]\left[\left[\begin{array}{cc}H_{22}^{I T h} & N_{22}^{I T h} \\ L_{22}^{I T h} & P_{22}^{I T h}\end{array}\right]+\left[\begin{array}{cc}H_{22}^{C} & N_{22}^{C} \\ L_{22}^{C} & P_{22}^{C}\end{array}\right]+\left[\begin{array}{cc}k_{x f}+i \omega c_{x f} & 0 \\ 0 & k_{\theta y M}+i \omega c_{\theta y M}\end{array}\right]^{-1}\right]^{-1}\left[\begin{array}{c}H_{23}^{T} \\ N_{23}^{T}\end{array}\right]$

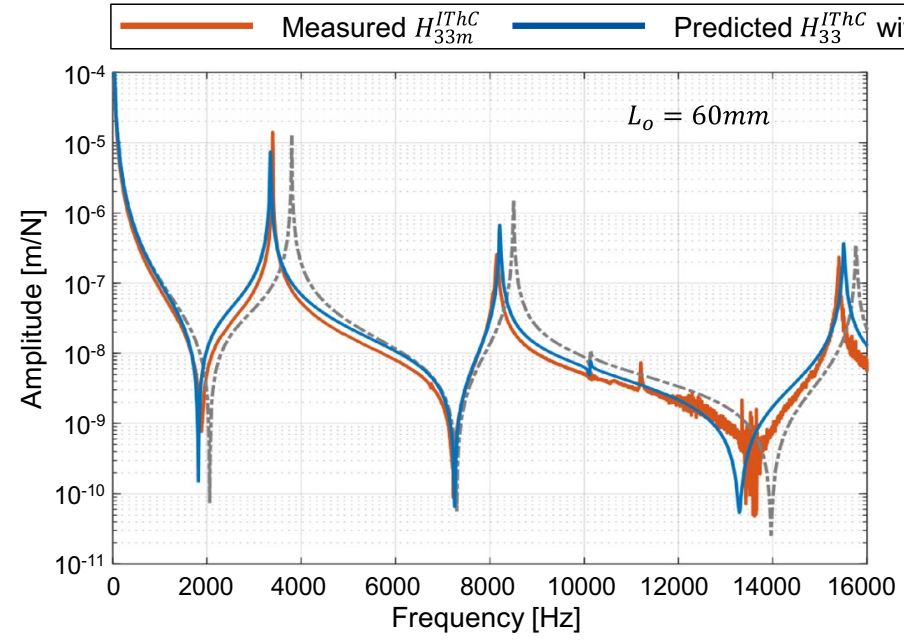

(a)

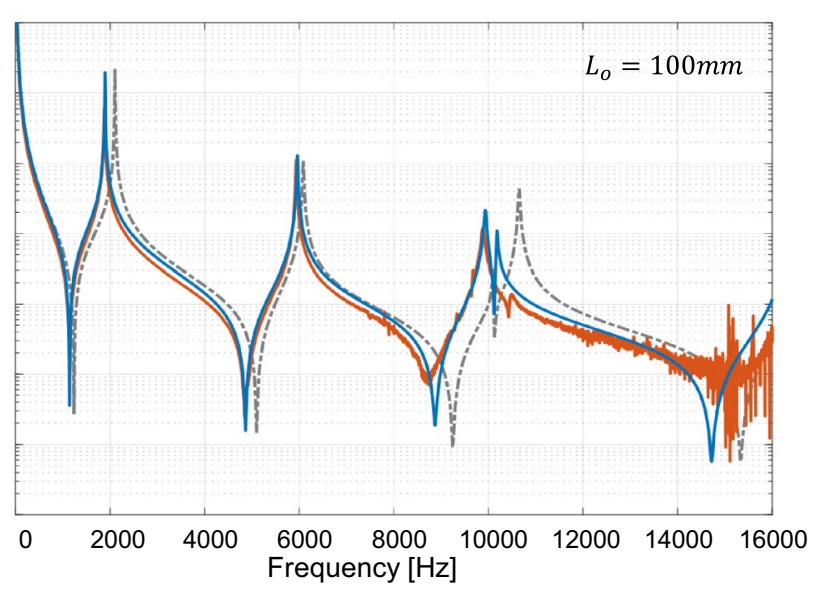

(b)

Fig. 3 Comparison of displacement to force FRFs at point $3 H_{33}^{I T h C}$, for carbide blank overhang (a) $L_{\mathrm{o}}=60 \mathrm{~mm}$ and (b) $L_{o}=100 \mathrm{~mm}$ 
shown in Fig. 2b. The subscript ' $m$ ' denotes an experimentally obtained FRF. The influence of sensor mass loading on the measured FRF was compensated using the structural modification method [12]. Figure 3 shows the measured and predicted tip FRFs for a tool holder with two $16 \mathrm{~mm}$ carbide blanks with overhangs of $60 \mathrm{~mm}$ and $100 \mathrm{~mm}$. The predicted and measured FRFs have similar courses, however, due to the missing joint parameters, the predicted FRF has resonance peaks located at relatively higher frequencies. The joint parameters can now be identified by comparing of both these FRFs. Literature provides several approaches for identifying joint parameters. Some of them are: manual or visual tuning of parameters [13, 21], parameter variation [1] and curve fitting or non-linear optimization $[9,15]$. The objective function for these approaches has been to reduce the difference between the predicted and measured amplitudes within a frequency range. For the freely-constrained case, a much better indicator of the influence of the joint stiffness is the location of the resonance peaks. Hence, in the proposed approach, the objective function is defined to reduce the weighted difference between the location (frequency) of the predicted and measured resonance peaks:

$$
\min _{K}\left(\sum_{i=1}^{n} W_{i}^{f} *\left|f_{i}^{m}-f_{i}\right|+W_{i}^{a b s} *\left\|H_{33, m}^{I T h C}-H_{33}^{I T h C}\right\|\right)
$$

where, $f_{i}^{m}$ corresponds to the frequency of the $i$-th resonance peak of the measurement and $f_{i}$ to that of the calculated peak. $W_{i}^{f}, W_{i}^{a b s}$ are the weightages assigned to the $i$-th frequency and absolute error respectively. Where, $W_{i}^{f} \gg W_{i}^{a b s}$ to enable a faster convergence of the optimization problem. A genetic algorithm was utilized to solve the optimization problem so that a global minimum to the bounded problem could be found. The identified joint parameters were used to elastically couple the interfaceholder assembly with the one segment beam representing the blank (Fig. 3). A very good correspondence can be observed between the measurements and the prediction with identified joint parameters. Both, the poles as well as the zeros could be matched well by the identified joint parameters. Due to the free-free condition, the structural modes of the tool assembly can be observed without the disturbing influence of surrounding structure. In addition, the use of acceleration sensor enables the reliable measurement till higher frequency $(16 \mathrm{kHz})$.

\subsection{Fluted segment modelling}

Once the joint properties for the holder-carbide blank have been identified, the carbide blank is replaced with the corresponding real tool with the same insertion length. The extended tool model is also modified by replacing the one-segment beam with the elastic coupling of a two-segment beam. The two segments are intended to represent the overhanging shank part of the real tool $\left(T_{\mathrm{s}}\right)$ and the fluted segment $\left(T_{\mathrm{f}}\right)$ respectively (Fig. 1b). Initially, both the beams possesses the same diameter of the shank. The shank segment $T_{\mathrm{s}}$ is first coupled elastically using the identified joint properties with the extended holder at point 2 giving the compliance matrix at the free-end of the shank as,

$\boldsymbol{G}_{44}^{I T h T_{s}}=\boldsymbol{G}_{44}^{T_{s}}+\boldsymbol{G}_{42}^{T_{s}}\left(\boldsymbol{G}_{22}^{T_{s}}+\boldsymbol{G}_{22}^{I T h}+\boldsymbol{K}^{-1}\right)^{-1} \boldsymbol{G}_{24}^{T_{s}}$

Applying the force equilibrium and compatibility conditions between the shank and the fluted segment at 4 , gives the expression for the direct compliance matrix of the complete tool $T$ at 4 in the rigidly coupled state,

$\boldsymbol{G}_{44}^{I T h T}=\boldsymbol{G}_{44}^{T_{f}}-\boldsymbol{G}_{44}^{T_{f}}\left(\boldsymbol{G}_{44}^{I_{f}}+\boldsymbol{G}_{44}^{I T h T_{s}}\right)^{-1} \boldsymbol{G}_{44}^{T_{f}}$

In this formulation, the compliance matrix $\boldsymbol{G}_{44}^{I T h T_{s}}$ is available from Eq. 7 and the reference displacement to force FRF $H_{44 m}^{I T h T}$ is obtained from a measurement of the freelyconstrained tool assembly (Fig. 2c). Here also, an acceleration sensor is used to allow reliable measurement till higher frequencies $(16 \mathrm{kHz})$ and its mass loading effect is compensated for. The unknown compliance matrix $\boldsymbol{G}_{44}^{T_{f}}$ in Eq. 8 depends on the geometric properties of the equivalent fluted segment beam. Since the reference FRF is available, the geometric parameter $\left(d_{\text {eff }}\right)$ can be varied until a good correspondence between the measured and predicted FRF is achieved. For this, a linear optimization algorithm, which minimizes an objective function similar to the one suggested in Eq. 6, is selected. The formulation in Eq. 8 is advantageous for this estimation because of the following reasons: (a) out of the four compliance matrices of the fluted segment beam $T_{f}$ only a single compliance matrix at 44 is required in the above expression. This greatly improves the efficiency of the optimization algorithm, as only the compliance matrix at one end needs to be calculated. (b) The measurement of $H_{44 m}^{I T h T}$ on the shank is more convenient than tap testing on the free end of the fluted segment. This is because of the difficulty of attaching sensors on the tool edges and the risk of damage to impact hammer. The measured and calculated (full cylinder) $H_{44}^{I T h T}$ FRFs for two real end-mill tools with tool diameter of $16 \mathrm{~mm}$ and overhang lengths of $58 \mathrm{~mm}$ $(Z=3)$ and $100 \mathrm{~mm}(Z=4)$ are shown in Fig. 4. The detailed description of these end mills can be found in Table 2. FEM simulations showed that the bending of the fluted section contributes significantly to the first mode shape. Therefore, the first resonance provides an excellent reference for identifying the equivalent effective diameter. The higher bending modes do not correspond to significant modal strains of the fluted segment alone. Hence, the correspondence of the predicted FRF with $d_{e f f}$ is much more important at the first 


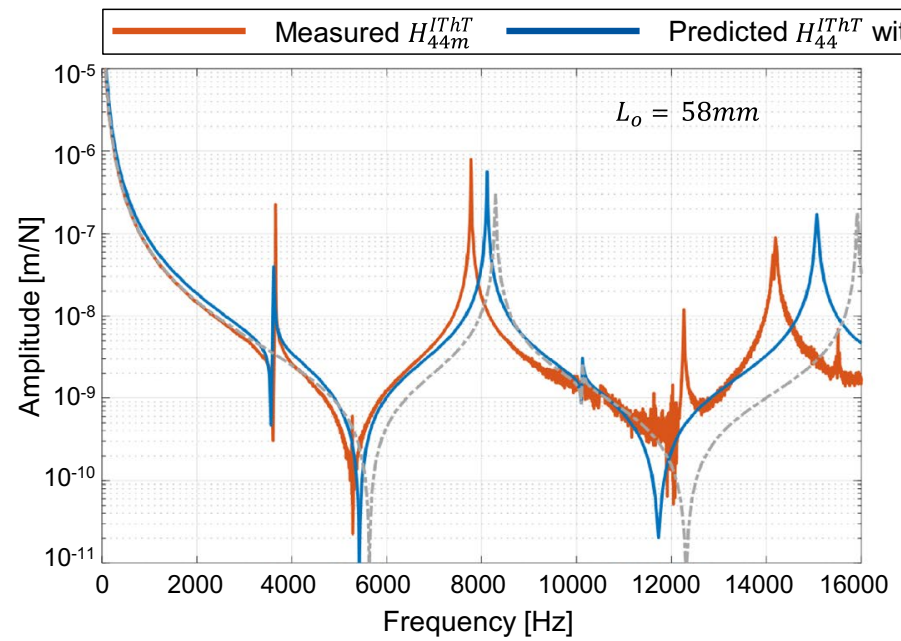

(a)

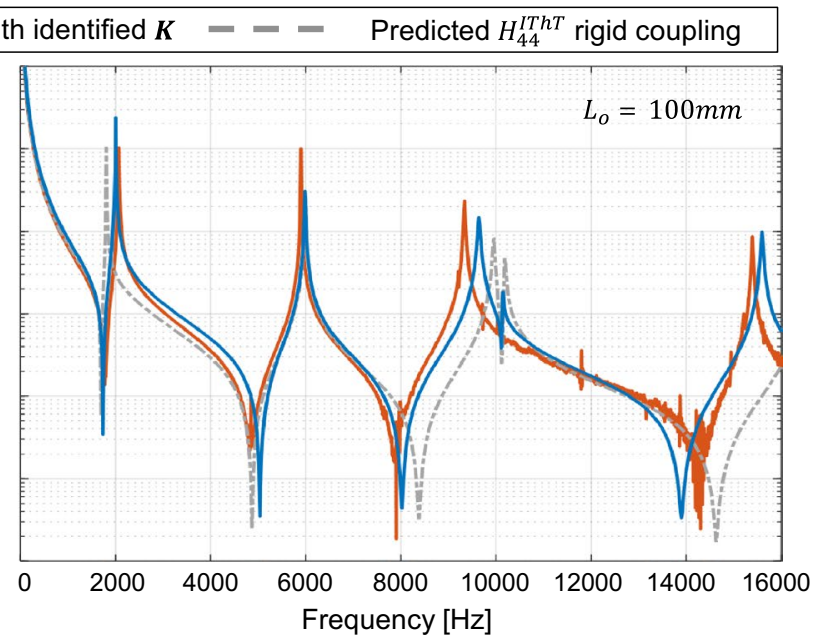

(b)

Fig. 4 Comparison of displacement to force FRFs at point $4 H_{44 m}^{I T h T}$, for end mill with overhang (a) $L_{\mathrm{o}}=58 \mathrm{~mm}, Z=3 \mathrm{and}(\mathbf{b}) L_{\mathrm{o}}=100 \mathrm{~mm}, Z=4$

resonance than the higher ones. It can be observed that the resonance frequency of the first bending mode for the full cylinders lies at a lower frequency while the other modes lie at higher frequencies compared to the measurement. The higher modal mass without proportional increase in modal stiffness associated with the full cylinder could explain the shift to lower frequency. The effective diameter estimated by the linear optimization algorithm is used to create the second beam of the two-segment beam. As a check, displacement to force FRFs $H_{44}^{I T h T}$ with the effective diameters are also plotted in Fig. 4. Here as well, a good correspondence between the measured and predicted FRFs can be observed. Especially the first bending mode could be represented accurately. Clearly, the effective diameter is a simplification of the complex tool geometry and cannot capture all dynamic effects corresponding to the higher modes. The following experimental implementation and validation shows that this simplification provides sufficiently accurate prediction without extensive modelling effort.

\section{Experimental implementation and validation}

The proposed method was implemented for a Haimer shrink fit tool holder (A63.140.16) with an internal diameter of $16 \mathrm{~mm}$ and an insertion depth of $50 \mathrm{~mm}$. It was simplified and divided in 6 cylindrical segments. Each segment was modelled as a Timoshenko beam and rigidly coupled with each other. The holder is equipped with four M5 $\times 8$ threaded balancing holes whose corresponding segment was assigned an outer diameter which produced the same area moment of inertia as the real cross section with the holes. The segments of the holder with the inserted carbide material was modelled as a composite beam where the physical properties were combined in ratio of their respective surface area [19]. The properties of holder material (Steel) and carbide blank were taken to be: Young's modulus $E_{\mathrm{s}}=2.069 * 10^{11} \mathrm{~N} / \mathrm{m}^{2}, E_{\mathrm{c}}=5.8 * 10^{11} \mathrm{~N} / \mathrm{m}^{2}$, density $\rho_{\mathrm{s}}=7829 \mathrm{~kg} / \mathrm{m}^{3}, \rho_{\mathrm{c}}=14550 \mathrm{~kg} / \mathrm{m}^{3} ;$ Poisson's ratio $v_{\mathrm{s}}=0.3$, $\nu_{\mathrm{c}}=0.22$ and structural damping loss factor of $\gamma_{\mathrm{s}}=0.0015$ ,$\gamma_{c}=0.002$. The holder has an HSK-A63 interface which was modelled as an FE part as described in Sect. 2. In the following sections, the ETMU approach was used for joint identification (Sect. 3.1) of carbide tools with different lengths as well as for estimating effective diameter of the fluted segment of three different end mills (Sect. 3.2). For validation, the updated tool models were coupled to the spindle-side compliance matrix $G_{11}^{S}$ of a 4-axis horizontal milling machine. The machine has working space diagonal of $1090 \mathrm{~mm}$ and is equipped with a $42 \mathrm{~kW}$ main spindle. The architecture corresponds to a cross-bed design. The spindle-side compliance matrix was obtained by decoupling or inverse receptance coupling of a cylindrical adapter.

\subsection{Joint Identification and analysis}

For the selected Haimer holder, six carbide blanks of $16 \mathrm{~mm}$ diameter and varying lengths of 100, 110, 120, 130, 140 and $150 \mathrm{~mm}$ were used. Utilizing the maximum insertion length of $50 \mathrm{~mm}$, gives overhang lengths $L_{o}$, from 50 to $100 \mathrm{~mm}$. The freely-constrained tool tip FRF $H_{33 m}^{I T h C}$ was measured for each holder-blank combination using the setup shown in Fig. 2b. The holder was suspended using elastic bands. A uniaxial piezoelectric charge accelerometer of type 4374 from Brüel \& Kjaer and an impulse hammer from Kistler 


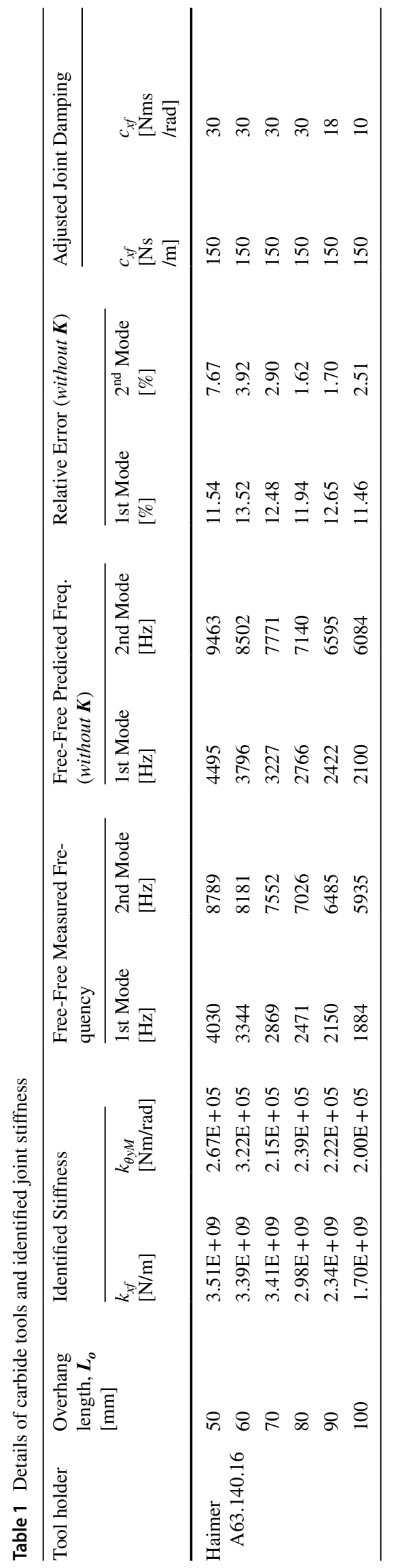

of type 9724A with a steel tip were used. An extended tool model was created for each holder-blank assembly and the tool tip FRF was first predicted by rigidly coupling the blank with the holder. Table 1 lists the used blanks with the corresponding measured and predicted (without joint parameters) resonance frequencies for the first and second modes. With increasing overhang, the relative difference between the resonance frequencies reduces (Fig. 5b). The results of the joint stiffness identification are also listed in Table 1 and represented graphically in Fig. 5a. Both translational $\left(k_{x f}\right)$ and rotational $\left(k_{\theta y M}\right)$ stiffness show a decreasing trend with increasing overhang. Between the minimum and maximum overhang, the identified translational stiffness reduces by $50.5 \%$ and rotational stiffness declines by $25 \%$, indicating that the location of the resonance frequency of the assembly is more sensitive to change in rotational than in translational stiffness. The decreasing trend corroborates well with the decreasing relative errors between the predicted (without $\boldsymbol{K})$ and measured resonance frequencies because a smaller magnitude of additional stiffness is required to compensate for a small difference in resonance frequencies. However, for the same insertion length, there is no physical change in the joint when the overhang is increased. Other complex joint models that consider the variation of contact pressure and stiffness along insertion length like the continuous uniform distributed spring-damper element model by Rezaei et. al [16] or the multipoint RSCA joint model by Schmitz et. al [21] do not show a sensitivity to overhang length. These models provide a more realistic representation. The variation of joint parameters for the same insertion length is probably due to the composite beam lumped spring-damper joint model, where the contact between the inserted tool and the holder is assumed to be rigid and the joint compliance is assumed to be acting at the interface between the holder and the overhanging tool. However this model can still accurately depict the joint behavior at different overhangs and is chosen in this work because of its simplicity and because the focus is on the method of identification. As mentioned previously, the proposed freely constrained approach can also be implemented to parameterize other joint models. The bounds for the optimization of stiffness terms were chosen to be $k_{x f} \in$ [1E7 1E10] and $k_{\theta y M} \in$ [1E4 1E7].

The identification of joint damping factor $c_{x f}, c_{\theta y M}$ in the freely constrained state still remains a challenge. It was observed that the amplitude of the resonance frequencies in this state is extremely sensitive to the quality of impact and measurement setup such that a consistent estimation of joint damping was not possible. Hence, literature values for joint damping [13] were used as starting values in this study and adjusted manually. Taking these values and the identified joint stiffness, the one-segment beam was elastically coupled with the holder. The holder-tool was subsequently coupled with the spindle-side compliance matrix to obtain 


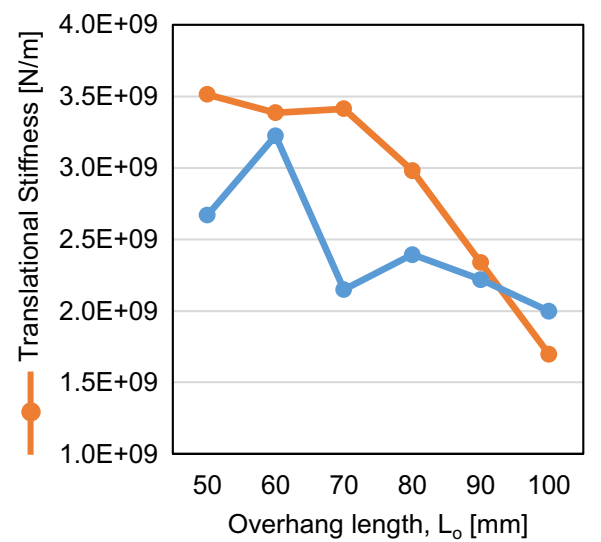

(a)
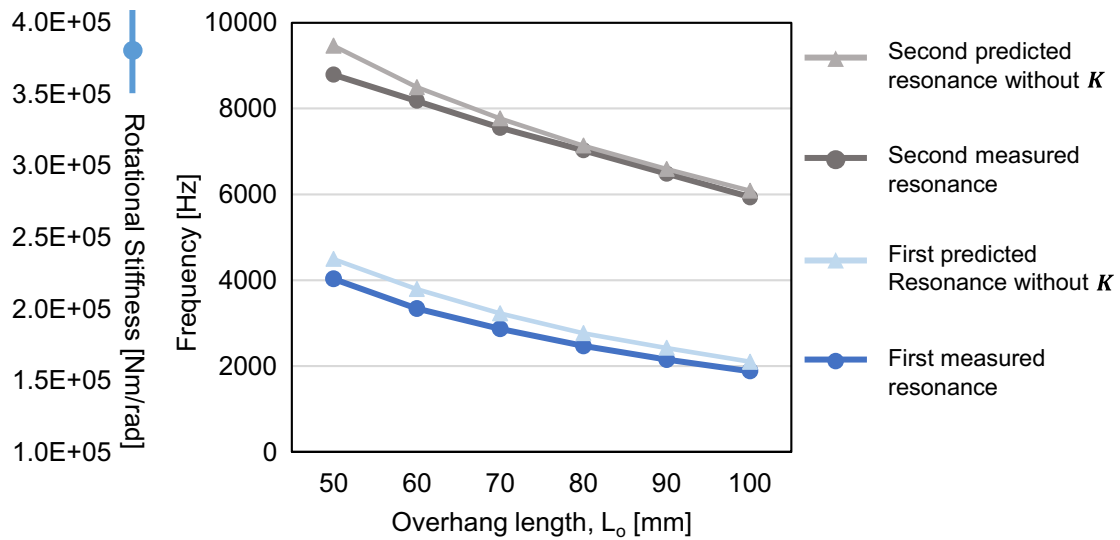

(b)

Fig. 5 Variation of (a) joint stiffness and (b) resonance frequencies with overhang length

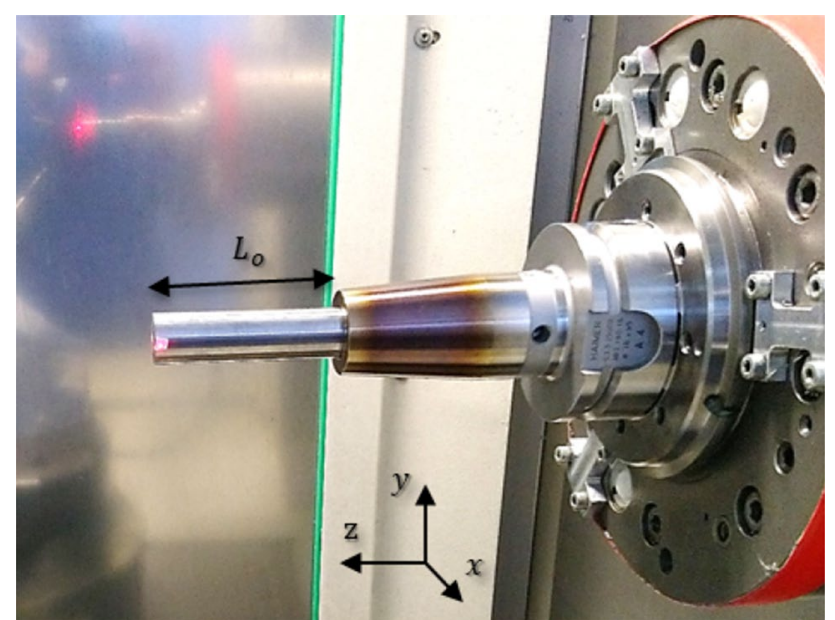

Fig. 6 Tool tip FRF measurement setup for $H_{33 m}^{S T h C}$ at 4-axis milling machine

the displacement to force tool-tip FRFs $H_{33}^{S T h C}$ in the clamped state in X-direction. For validation, corresponding reference FRFs $H_{33 m}^{S T h C}$ were measured with the help of a single-beam laser Doppler vibrometer and the impulse hammer (Fig. 6).

Figure 7 shows the comparison of the amplitude and phase diagrams for the predicted and measured tip FRFs. A very good correspondence could be achieved between the two for all overhang lengths indicating an appropriate identification of the joint stiffness. The adjusted damping is also listed in Table 1 . Only for the $50 \mathrm{~mm}$ and $60 \mathrm{~mm}$ overhang, a certain amplitude error is observed in the clamped-free state. Since the free-free comparison for these tools show a good fit (Fig. 3), it can be assumed that the joint parameters could be identified correctly. The cause for the deviation is probably the composite beam and lumped spring-damper model, which perhaps cannot alone compensate for the deviation between rigidly coupled and measured free-free behavior for short tools.

\subsection{Fluted segment estimation}

Three carbide end mills with different lengths, diameters, number of teeth, fluted lengths and helix angles were chosen for the validation of the estimation of the effective diameter. The details of the used holders and tools are listed in Table 2. The joint identification for these tool lengths and holders was carried out with carbide blanks as explained in Sect. 2.1. Effective diameter of the fluted segment for the each tool was estimated based on the measured FRF $H_{44 m}^{I T h T}$ of the extended tool assembly as shown in Sect. 2.2. The analysis of each end mill is done on a case-by-case basis.

\subsubsection{Case A: $16 \mathrm{~mm}$ end mill with $Z=3$}

A three-fluted end mill with a tool and shank diameter of $16 \mathrm{~mm}$ and a total length of $108 \mathrm{~mm}$ was inserted fully in a Haimer shrink-fit tool holder. For the measurement of the reference FRF, this tool assembly was clamped in the main spindle of the 4-axis milling machine and the tip FRF in X-direction was obtained using the vibrometer and the impulse hammer. The joint properties for this tool were taken to be those identified for the carbide blank of $60 \mathrm{~mm}$. The freely constrained direct FRF at point $4 H_{44 m}^{I T h T}$ along with the analytical FRF $H_{44}^{I T h T}$ with the estimated effective diameter of $d_{\text {eff }}=11.8 \mathrm{~mm}$ show a good correspondence (Fig. 4a). Using this tool model, the tool tip FRF $H_{33}^{S T h T}$ in clamped state was predicted and compared with the reference in Fig. 8. The eigenfrequency of the tool bending mode as well as the quasi-static compliance could be predicted well. A certain error in the amplitude of the tool mode was 

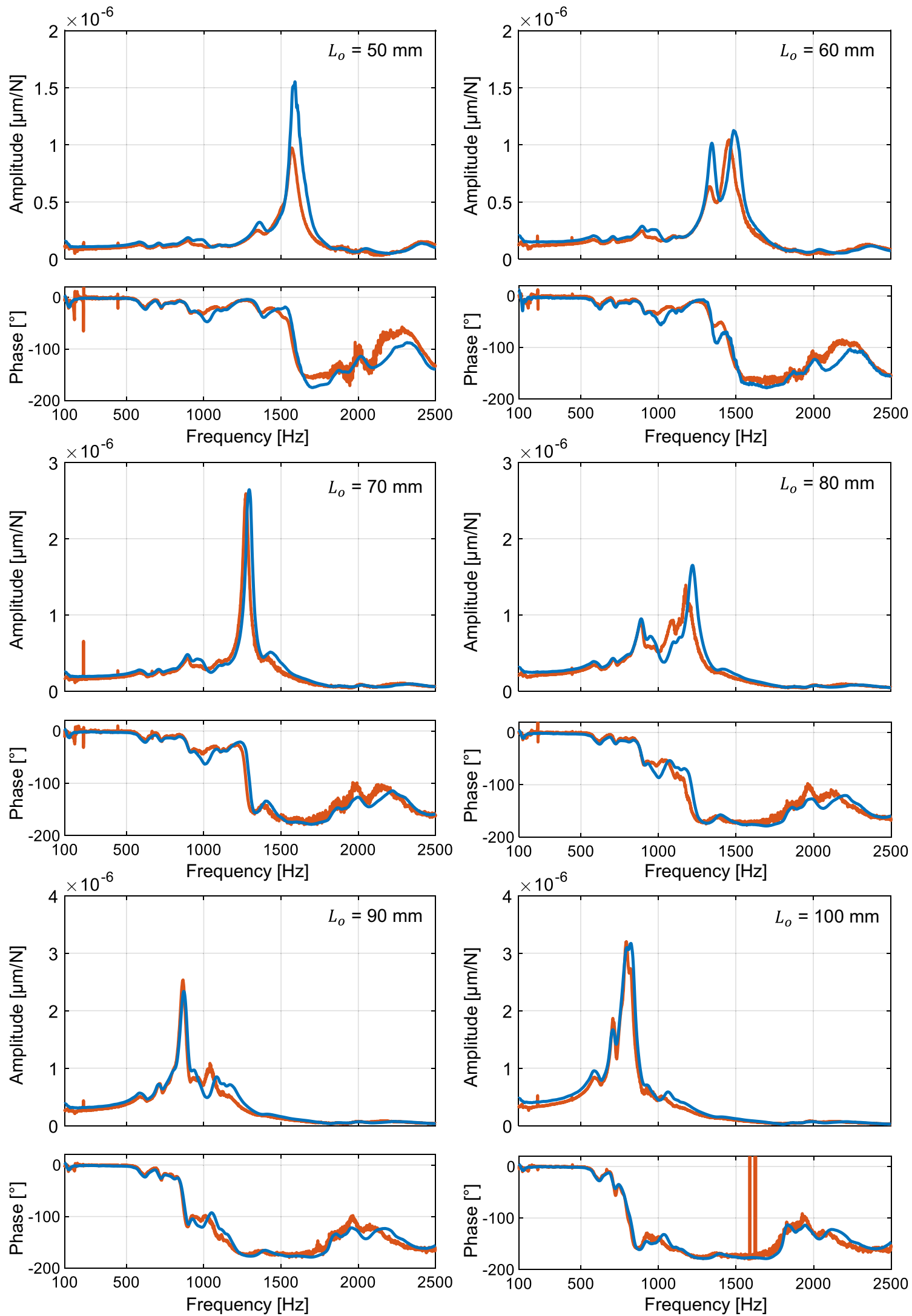

Measurement $=$ Prediction

Fig. 7 Comparison of measured tool-tip FRFs with predicted FRFs using ETMU 
Table 2 Details of end mills used for validation and estimated effective fluted segment diameter

\begin{tabular}{llllllllll}
\hline Tool Holder & Cases & $\begin{array}{l}\text { Overhang } \\
\text { length } \\
{[\mathrm{mm}]}\end{array}$ & No. of teeth & $\begin{array}{l}\text { Helix angle } \\
{\left[{ }^{\circ}\right]}\end{array}$ & $\begin{array}{l}\text { Shank } \\
\text { diameter } \\
{[\mathrm{mm}]}\end{array}$ & $\begin{array}{l}\text { Tool } \\
\text { diameter } \\
{[\mathrm{mm}]}\end{array}$ & $\begin{array}{l}\text { Effective } \\
\text { diameter } \\
{[\mathrm{mm}]}\end{array}$ & $\begin{array}{l}\text { Overhang } \\
\text { shank length } \\
{[\mathrm{mm}]}\end{array}$ & $\begin{array}{l}\text { Fluted seg- } \\
\text { ment length } \\
{[\mathrm{mm}]}\end{array}$ \\
\hline Haimer A63.140.16 & Case A & 58 & 3 & 45 & 15.5 & 16 & 11.8 & 15 & 43 \\
& Case B & 100 & 4 & 38 & 15.5 & 16 & 12.4 & 59 & 41 \\
Haimer A63.140.10 & Case C & 58 & 2 & 45 & 9.4 & 10 & 6.22 & 27 & 31 \\
\hline
\end{tabular}
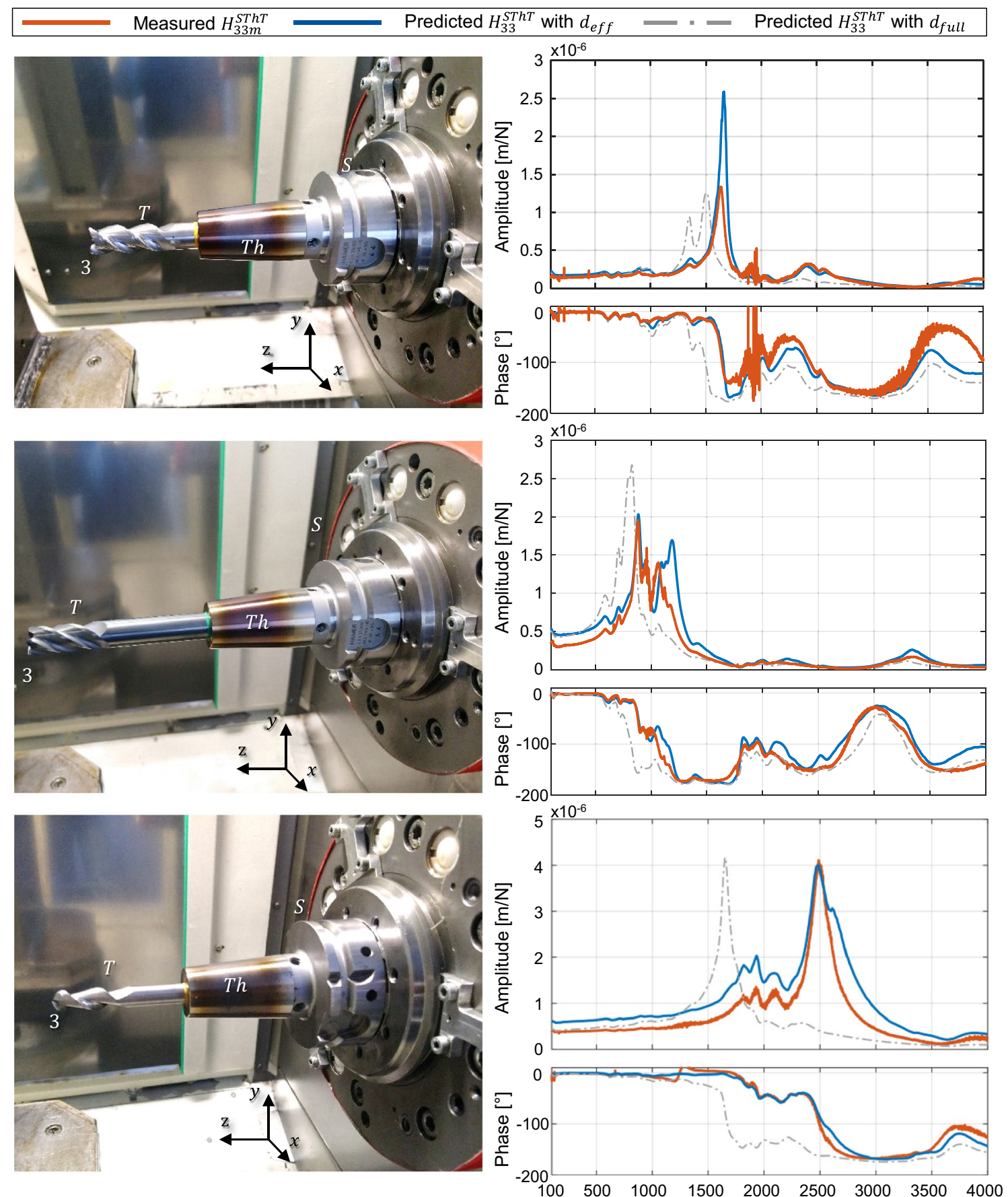

Fig. 8 Comparison of tool tip FRFs in X-direction for Case A (top), Case B (middle) and Case C (bottom) 
Table 3 Identified joint properties of $10 \mathrm{~mm}$ diameter blank and tool holder

\begin{tabular}{|c|c|c|c|c|c|c|}
\hline \multirow[t]{2}{*}{ Tool holder } & \multirow[t]{2}{*}{ Blank diameter $[\mathrm{mm}]$} & \multirow{2}{*}{$\begin{array}{l}\text { Overhang length, } \boldsymbol{L}_{\boldsymbol{o}} \\
{[\mathrm{mm}]}\end{array}$} & \multicolumn{2}{|c|}{ Identified stiffness } & \multicolumn{2}{|c|}{ Adjusted joint damping } \\
\hline & & & $\begin{array}{l}k_{x f} \\
{[\mathrm{~N} / \mathrm{m}]}\end{array}$ & $\begin{array}{l}k_{\theta y M} \\
{[\mathrm{Nm} / \mathrm{rad}]}\end{array}$ & $\begin{array}{l}k_{x f} \\
{[\mathrm{Ns}} \\
/ \mathrm{m}]\end{array}$ & $\begin{array}{l}k_{x f} \\
{[\mathrm{Nms}} \\
/ \mathrm{rad}]\end{array}$ \\
\hline Haimer A63.140.10 & 10 & 58 & $8.43 E+08$ & $3.85 E+04$ & 1 & 0.5 \\
\hline
\end{tabular}

observed which could result from not considering the exact geometry of the flutes combined with the joint modelling error. Additionally, the tip FRF with a full cylinder of tool diameter $d_{\text {full }}=16 \mathrm{~mm}$ to demonstrate the error associated with ignoring the fluted segment modelling.

\subsection{Case B: $16 \mathrm{~mm}$ end mill with $Z=4$}

A long four-fluted end mill with $16 \mathrm{~mm}$ diameter with a total length of $150 \mathrm{~mm}$ was inserted in the same shrink-fit holder with full insertion. The joint parameters of the carbide tool with the same total length were used for the tool model. An effective diameter of $12.4 \mathrm{~mm}$ was estimated using the freely constrained FRFs shown in Fig. 4b. The comparison of the predicted and measured tool tip FRF $H_{33}^{S T h T}$ is provided in Fig. 8. Although a certain deviation is observed at around $1120 \mathrm{~Hz}$, the dominant tool mode as well as the quasi-static stiffness could be estimated with sufficient accuracy. A rough estimation of these aspects without extensive modelling is sufficient for predicting the surface location error due to tool deflection and for avoiding excitation of dominant resonance frequencies through process excitation.

\subsection{Case $C: 10 \mathrm{~mm}$ end mill with $Z=2$}

As further validation, a Haimer tool holder with $10 \mathrm{~mm}$ inner diameter and a two-fluted rounded end mill of total length of $108 \mathrm{~mm}$ was taken. The holder was modelled analytically using six Timoshenko beam segments. The results of the joint identification with a fully inserted carbide blank are listed in Table 3. Compared to the $16 \mathrm{~mm}$ holder this joint has roughly a factor 10 lower stiffness values. The asymmetric geometry of two-fluted segment results in variation of dynamics with angle of rotation of the end mill. However, for this tool no significant variation in dynamics was observed. This can be attributed to the shorter fluted length compared to the overhang length and corresponds to the observations in [13]. The comparison of the predicted and measured tool tip FRF $H_{33}^{S T h T}$ shows that the tool mode could be predicted well. Considering the fluted segment as a full cylinder resulted in a poor prediction for all end mills.

\section{Conclusions}

This paper proposes the extension of the beam model of the holder and tool or blank such that this model can be updated using experimentally obtained FRFs of the tool assembly in freely constrained state. In this way, different unknown parameters can be identified accurately and without blocking the machine tool. The extended tool model updating approach was implemented first for joint identification of a $16 \mathrm{~mm}$ thermal shrink-fit holder with different carbide blank lengths. The prediction of tip FRF of the extended model with the rigid coupling of the one-segment beam lead to resonance frequencies, which were slightly higher than the measured resonance frequencies. This difference was a reliable indicator of the joint stiffness such that elastic coupling with the identified stiffness resulted in a good conformance with the measured free-free FRF up to $16 \mathrm{kHz}$. The validation by comparing the measured and analytical tip FRF of the holder-blank assembly in a machine spindle confirmed that the stiffness values were correctly identified. Similarly, the comparison of freely constrained FRFs between the shank and flutes (at point 4) showed that an effective diameter of fluted segment could appropriately describe the dynamic behavior of the real tool for the first modes. The choice of point 4 , for comparing the dynamics, greatly facilitates the estimation problem. The tool tip FRF could be predicted with reasonable accuracy for different tool diameters, fluted lengths, helix angels and number of teeth. This rough prediction can be used for process planning by avoiding the excitation of critical chatter frequencies. If a more accurate prediction of stability boundaries is required, a more detailed flute model is necessary. Hence, a sufficiently accurate model of the holder-tool assembly with only two offline reference measurements was achieved, thereby increasing the efficiency of the tool modelling process.

The achieved results show that a good conformance of the dynamic behavior of the extended tool model with the real assembly is a good indicator of the correctness of the analytical model. This creates the possibility of completely offline validation of other aspects of tool modelling such as the discretization required for representing tapered holder segments, approach for modelling composite beams and other features. 
Acknowledgements The authors wish to gratefully acknowledge the support of the German Research Foundation (Deutsche Forschungsgemeinschaft, DFG). This work was funded as part of the DFG Project 'Experimental substructure coupling for vibration analysis in machine tools' (Project Number-BR 2905/55-2).

Funding Open Access funding enabled and organized by Projekt DEAL.

Open Access This article is licensed under a Creative Commons Attribution 4.0 International License, which permits use, sharing, adaptation, distribution and reproduction in any medium or format, as long as you give appropriate credit to the original author(s) and the source, provide a link to the Creative Commons licence, and indicate if changes were made. The images or other third party material in this article are included in the article's Creative Commons licence, unless indicated otherwise in a credit line to the material. If material is not included in the article's Creative Commons licence and your intended use is not permitted by statutory regulation or exceeds the permitted use, you will need to obtain permission directly from the copyright holder. To view a copy of this licence, visit http://creativecommons.org/licenses/by/4.0/.

\section{References}

1. Ahmadi K, Ahmadian H (2007) Modelling machine tool dynamics using a distributed parameter tool-holder joint interface. Int J Mach Tools Manuf. 47(12-13):1916-1928

2. Aristizabal-Ochoa JD (2004) Timoshenko beam-column with generalized end conditions and nonclassical modes of vibration of shear beams. J EngMech 130(10):1151-1159

3. Brecher C, Weck M (2017) Werkzeugmaschinen Fertigungssysteme. Konstruktion, Berechnung und messtechnische Beurteilung. Berlin, Heidelberg: Springer Berlin Heidelberg.

4. Ertürk A, Özgüven HN, Budak E (2006) Analytical modeling of spindle-tool dynamics on machine tools using Timoshenko beam model and receptance coupling for the prediction of tool point FRF. Int J Mach Tools Manuf. 46(15):1901-1912

5. Filiz S, Cheng C-H, Powell KB, Schmitz TL, Ozdoganlar OB (2009) An improved tool-holder model for RCSA tool-point frequency response prediction. Precision Eng 33(1):26-36

6. Kivanc EB, Budak E (2004) Structural modeling of end mills for form error and stability analysis. Int J Mach Tools Manuf 44(11):1151-1161

7. Kops L, Vo DT (1990) Determination of the equivalent diameter of an end mill based on its compliance. CIRP Ann 39(1):93-96

8. Liao J, Zhang J, Feng P, Yu D, Wu Z (2017) Identification of contact stiffness of shrink-fit tool-holder joint based on fractal theory. Int J AdvManufTechnol 90(5-8):2173-2184
9. Matthias W, Özşahin O, Altintas Y, Denkena B (2016) Receptance coupling based algorithm for the identification of contact parameters at holder-tool interface. CIRP J ManufSci Technol. 13:37-45

10. Namazi M, Altintas Y, Abe T, Rajapakse N (2007) Modeling and identification of tool holder-spindle interface dynamics. Int J Mach Tools Manuf. 47(9):1333-1341

11. Özşahin O, Ertürk A, Özgüven HN, Budak E (2009) A closedform approach for identification of dynamical contact parameters in spindle-holder-tool assemblies. Int J Mach Tools Manuf. 49(1):25-35

12. Özşahin O, Özgüven HN, Budak E (2010) Analysis and compensation of mass loading effect of accelerometers on tool point FRF measurements for chatter stability predictions. Int J Mach Tools Manuf. 50(6):585-589

13. Özşahin O, Altintas Y (2015) Prediction of frequency response function (FRF) of asymmetric tools from the analytical coupling of spindle and beam models of holder and tool. Int J Mach Tools Manuf. 92:31-40

14. Park SS, Altintas Y, Movahhedy M (2003) Receptance coupling for end mills. Int J Mach Tools Manuf. 43(9):889-896

15. Park SS, Chae J (2008) Joint identification of modular tools using a novel receptance coupling method. Int J AdvManuf Technol. 35(11-12):1251-1262

16. Rezaei MM, Movahhedy MR, Moradi H, Ahmadian MT (2012) Extending the inverse receptance coupling method for prediction of tool-holder joint dynamics in milling. J Manuf Processes. 14(3):199-207

17. Schmitz TL, Donalson RR (2000) Predicting high-speed machining dynamics by substructure analysis. CIRP Ann 49(1):303-308

18. Schmitz TL, Duncan GS (2005) Three-component receptance coupling substructure analysis for tool point dynamics prediction. $\mathrm{J}$ ManufSciEng 127(4):781

19. Schmitz TL, Duncan GS (2006) Receptance coupling for dynamics prediction of assemblies with coincident neutral axes. J Sound Vib 289(4-5):1045-1065

20. Schmitz TL, Powell K, Won D, Scott Duncan G, Gregory Sawyer W, Ziegert JC (2007) Shrink fit tool holder connection stiffness/ damping modeling for frequency response prediction in milling. Int J Mach Tools Manuf 47(9):1368-1380

21. Schmitz T, Honeycutt A, Gomez M, Stokes M, Betters E (2019) Multi-point coupling for tool point receptance prediction. J Manuf Process 43:2-11

Publisher's Note Springer Nature remains neutral with regard to jurisdictional claims in published maps and institutional affiliations. 